\title{
A REMARK ON $k$ TH-ORDER LINEAR FUNCTIONAL EQUATIONS WITH CONSTANT COEFFICIENTS
}

\author{
JITKA LAITOCHOVÁ \\ Received 30 January 2006; Accepted 18 May 2006
}

\begin{abstract}
Abel functional equations are associated to a linear homogeneous functional equation with constant coefficients. The work uses the space $S$ of continuous strictly monotonic functions. Generalized terms are used, because of the space $S$, like composite function, iterates of a function, Abel functional equation, and linear homogeneous functional equation in $S$ with constant coefficients. The classical theory of linear homogeneous functional and difference equations is obtained as a special case of the theory in space $S$. Equivalence of points and orbits of a point are introduced to show the connection between the linear functional and the linear difference equations in $S$. Asymptotic behavior at infinity is studied for a solution of the linear functional equation.
\end{abstract}

Copyright (c) 2006 Jitka Laitochová. This is an open access article distributed under the Creative Commons Attribution License, which permits unrestricted use, distribution, and reproduction in any medium, provided the original work is properly cited.

\section{Introduction}

The linear functional equations are considered in the space of real-valued functions of a real variable $x \in \mathscr{F}, \mathscr{F}=(-\infty, \infty)$. The set $\mathbb{N}$ denotes the set of positive integers. The set $\mathbb{Z}$ denotes integers, and the set $\mathbb{R}$ denotes real numbers. Symbol $C_{0}(\mathscr{F})$ is the set of continuous functions on the interval $\mathscr{F}$.

Definitions of the terms which we generalize in the space $S$ can be found in [1-5]. In particular, we generalize the notions of iterates and linear homogeneous functional equations with constant coefficients.

1.1. Definition of the space $S$. A function $f \in C_{0}(\mathscr{F})$ belongs to $S$ if and only if it maps the interval $\mathscr{f}$ one-to-one onto the interval $(a, b)$, where $a \in \mathbb{R}$ or $a=-\infty, b \in \mathbb{R}$ or $b=\infty$.

1.2. Multiplication in $S$. Let us choose in $S$ an arbitrary function $X$, a so-called canonical function, and let $X^{*}$ be the inverse function to $X$. Let $F, G \in S$. The composite function $H=F X^{*} G(x)$ will be called a product and denoted by $\mathbf{H}=\mathbf{F} \circ \mathbf{G}$. 
It is easy to show that $H \in S$. The set $S$ with the operation of multiplication $\circ$ forms a noncommutative group $\mathscr{G}$, where the canonical function $X$ is the neutral element. Further, to each element $F \in S$ there is an inverse element $\mathbf{F}^{-1}=X F^{*} X$ in $\mathscr{G}$, where $F^{*} \in S$ is the inverse function to $F \in S$.

Boldface promotion of symbols will be used to denote elements of $\mathscr{G}$. Then $F, G \in S$ correspond to $\mathbf{F}, \mathbf{G} \in \mathscr{G}$ and $\mathscr{G}$-multiplication is defined by composition:

$$
\mathbf{F} \circ \mathbf{G}(x) \equiv F\left(X^{*}(G(x))\right) .
$$

If $f \in C_{0}(\mathscr{F}), \Phi \in S$, then the product $f \circ \Phi(x)$ (not necessarily in $\mathscr{G}$ ) is defined to be the composite function $f\left(X^{*}(\Phi(x))\right) \in C_{0}(\mathscr{F})$. In general, $f$ is not one-to-one; boldface promotion of $f$ is disallowed, because $f \notin S$.

1.3. Iteration in $S$. Let $X \in S$ be the canonical function. Let $\Phi \in S$. The iterates of the function $\Phi$ in $S$ are given by group operations as follows:

$$
\begin{gathered}
\boldsymbol{\Phi}^{0}(x)=X(x), \\
\boldsymbol{\Phi}^{n+1}(x)=\boldsymbol{\Phi} \circ \boldsymbol{\Phi}^{n}(x), \quad x \in \mathscr{F}, n=0,1,2, \ldots, \\
\boldsymbol{\Phi}^{n-1}(x)=\boldsymbol{\Phi}^{-1} \circ \boldsymbol{\Phi}^{n}(x), \quad x \in \mathscr{J}, n=0,-1,-2, \ldots,
\end{gathered}
$$

where $\Phi^{-1}$ is the inverse element to the element $\Phi$ in $S$ according to multiplication $\circ$ in group $\mathscr{G}$.

\section{Linear functional equations of the $k$ th order with constant coefficients}

Let $a_{j} \in \mathbb{R}, j=0,1,2, \ldots, k$. Then the equation

$$
a_{k} f \circ \boldsymbol{\Phi}^{k}(x)+a_{k-1} f \circ \boldsymbol{\Phi}^{k-1}(x)+\cdots+a_{1} f \circ \boldsymbol{\Phi}^{1}(x)+a_{0} f \circ \boldsymbol{\Phi}^{0}(x)=0
$$

in $S$ is called a linear homogeneous functional equation of $k$ th order with constant coefficients. The coefficients are the constants $a_{j}, j=0,1,2, \ldots, k$. It is assumed that $a_{k} \neq 0$. A solution of $(2.1)$ is a function $f \in C_{0}(\mathscr{F})$ that satisfies the equation for all $x$. It is not assumed that $f$ is one-to-one.

Let $g(x)=X(x+1)$ and let $\Phi \in S$. The generalized Abel functional equation

$$
\boldsymbol{\alpha} \circ \Phi(x)=\mathbf{g} \circ \boldsymbol{\alpha}(x)
$$

is called the associated functional equation to (2.1). A solution of (2.2) is a function $\alpha \in S$ such that (2.2) holds for all $x$.

The algebraic equation

$$
a_{k} \lambda^{k}+a_{k-1} \lambda^{k-1}+\cdots+a_{1} \lambda+a_{0}=0
$$

is called the characteristic equation of (2.1). The left side of (2.3) is called the characteristic polynomial of (2.1). 
THeORem 2.1. Let (2.1) be given. Let $\lambda_{1}, \lambda_{2}, \ldots, \lambda_{k}$ be simple positive roots of the characteristic equation (2.3). Let $\alpha$ be a continuous solution of the associated Abel functional equation (2.2). Then the functions

$$
f_{1}=\lambda_{1}^{X^{*} \alpha(x)}, \quad f_{2}=\lambda_{2}^{X^{*} \alpha(x)}, \ldots, f_{k}=\lambda_{k}^{X^{*} \alpha(x)},
$$

are linearly independent solutions of (2.1).

Proof. Functions (2.4) have the form

$$
f=\lambda^{X^{*} \alpha(x)}
$$

where $\alpha \in S$ is a solution of the Abel equation (2.2) and $X^{*} \alpha(x)$ denotes the composite function $X^{*}(\alpha(x))$. Substitute (2.5) into (2.1), then

$$
\sum_{n=0}^{k} a_{n} \lambda^{X^{*} \alpha \circ \Phi^{n}(x)}=0 .
$$

Abel functional equation (2.2) implies

$$
X^{*} \alpha \circ \Phi^{n}(x)=X^{*} X\left(X^{*} \alpha(x)+n\right)=X^{*} \alpha(x)+n,
$$

$n=0,1,2, \ldots, k$, and then

$$
\lambda^{X^{*} \alpha(x)}\left[\sum_{n=0}^{k} a_{n} \lambda^{n}\right]=0 .
$$

Thus the function (2.5) is a solution of (2.1) if $\lambda$ is a root of the characteristic equation (2.3). Roots of the characteristic equation have to be positive for the functions (2.5) to be defined.

To show the solutions (2.4) are linearly independent, consider a linear combination $c_{1} f_{1}(x)+\cdots+c_{k} f_{k}(x)=0$ for all $x$. Choose samples $x=X^{*}\left(x_{0}\right), x=X^{*}\left(\Phi\left(x_{0}\right)\right), x=$ $X^{*}\left(\Phi^{2}\left(x_{0}\right)\right)$, and so forth, and form the matrix equation $A \mathbf{c}=\mathbf{0}$ for the coefficients $\mathbf{c}$, using the sampling matrix

$$
A=\left(\begin{array}{cccc}
f_{1} \circ \Phi^{0}\left(x_{0}\right) & f_{2} \circ \Phi^{0}\left(x_{0}\right) & \cdots & f_{k} \circ \Phi^{0}\left(x_{0}\right) \\
f_{1} \circ \Phi^{1}\left(x_{0}\right) & f_{2} \circ \Phi^{1}\left(x_{0}\right) & \cdots & f_{k} \circ \Phi^{1}\left(x_{0}\right) \\
\vdots & \vdots & \vdots & \vdots \\
f_{1} \circ \Phi^{k-1}\left(x_{0}\right) & f_{2} \circ \Phi^{k-1}\left(x_{0}\right) & \cdots & f_{k} \circ \Phi^{k-1}\left(x_{0}\right)
\end{array}\right) .
$$

Abel equation can be used to show that $\operatorname{det}(A) \neq 0$ for any $x_{0}$ in $\mathscr{F}$. The idea is born from the relation $f_{r} \circ \boldsymbol{\Phi}^{n}(x)=f_{r}(x) \lambda_{r}^{n}$ and the theory of Vandermonde determinants. Therefore, $\mathbf{c}=\mathbf{0}$ and the functions are independent. The proof is complete.

Automorphic functions. A function $p \in C_{0}(\mathscr{F})$ is called automorphic over $\Phi$ provided $p=p \circ \boldsymbol{\Phi}$. While constant functions are automorphic with respect to any $\Phi$, there are examples of nonconstant automorphic functions. One such is $p(x)=\sin (\pi(x-n))$ on 
4 A remark on linear functional equations

$n \leq x<n+1$, which satisfies $p(x)=p(x+1)$ or $p=p \circ \Phi$ for $\Phi(x)=x+1$. Others can be constructed from this example.

THeORem 2.2. Let (2.1) be given having solution $f_{0}$. Let $p$ be a continuous automorphic function, $p=p \circ \Phi$. Then $f(x)=p(x) f_{0}(x)$ is also a solution of $(2.1)$.

A more general solution is of the form

$$
c_{1} f_{1}+c_{2} f_{2}+\cdots+c_{k} f_{k}
$$

where $c_{1}, c_{2}, \ldots, c_{k}$ are automorphic functions over $\Phi$ and $f_{1}, \ldots, f_{k}$ are solutions of (2.1).

Proof. The details to show $f_{0}$ satisfies (2.1) are as follows:

$$
\begin{aligned}
\sum_{n=0}^{k} a_{n}\left(p f_{0}\right) \circ \boldsymbol{\Phi}^{n}(x) & =\sum_{n=0}^{k} a_{n}\left(p \circ \boldsymbol{\Phi}^{n}(x)\right)\left(f_{0} \circ \boldsymbol{\Phi}^{n}(x)\right) \\
& =\sum_{n=0}^{k} a_{n}(p(x))\left(f_{0} \circ \boldsymbol{\Phi}^{n}(x)\right) \\
& =p(x) \sum_{n=0}^{k} a_{n}\left(f_{0} \circ \boldsymbol{\Phi}^{n}(x)\right) \\
& =0 .
\end{aligned}
$$

The proof is complete.

THeorem 2.3. Let (2.1) be given. Let $\lambda_{0}$ be a positive real root of characteristic equation (2.3), of multiplicity $s, 1 \leq s \leq k$. Let $\alpha(x)$ be a continuous solution of Abel functional equation (2.2) and let $X^{*}$ be the inverse function to canonical function $X$. Then the functions

$$
f_{r}=\left(X^{*} \alpha(x)\right)^{r} \lambda_{0}^{X^{*} \alpha(x)}, \quad 0 \leq r<s
$$

are independent solutions of (2.1).

Proof. Let $p(\lambda)$ denote the characteristic polynomial. Assume for the first part of the proof that $X$ is the identity map. Define $f(x)=(\alpha(x))^{r} \lambda_{0}^{\alpha(x)}$. The following lemmas will be applied to complete the proof.

LeMmA 2.4. If $L=\lambda(d / d \lambda)$, then $L^{q}(p(\lambda))=\sum_{n=0}^{k} n^{q} a_{n} \lambda^{n}$ for $q \geq 0$.

Lemma 2.5. If $\lambda=\lambda_{0}$ and $0 \leq m \leq r<s$, then $\sum_{n=0}^{k} n^{r-m} a_{n} \lambda^{n}=0$. 
Lemma 2.6. $\left\{(\alpha(x))^{r} \lambda_{0}^{\alpha(x)}\right\}_{r=0}^{s-1}$ are independent if and only if the powers $\left\{(\alpha(x))^{r}\right\}_{r=0}^{s-1}$ are independent.

Lemma 2.7. Let $t_{n}=\alpha\left(x_{0}\right)+n$. Define matrix

$$
A=\left(\begin{array}{cccc}
1 & t_{1} & \cdots & t_{1}^{s-1} \\
1 & t_{2} & \cdots & t_{1}^{s-1} \\
\vdots & \vdots & \vdots & \vdots \\
1 & t_{s} & \cdots & t_{s}^{s-1}
\end{array}\right) .
$$

Then $\operatorname{det}(A) \neq 0$ and the powers of Lemma 2.6 are independent.

The proofs of the lemmas: for Lemma 2.4 use induction on $q$. For Lemma 2.5 the left side of the equation is $L^{q}(p(\lambda))$ for $q=r-m \geq 0$. Apply Lemma 2.4. Expand $L^{q}(p(\lambda))$ by calculus to verify that being zero at $\lambda=\lambda_{0}$ is possible because the derivatives $(d / d \lambda)^{n} p(\lambda)$ are zero at $\lambda=\lambda_{0}$ for $0 \leq r<s$, due to multiplicity of the root $\lambda_{0}$. For Lemma 2.6 write down a linear combination equal to zero. Cancel $\lambda_{0}^{\alpha(x)}$. For Lemma 2.7 the determinant is a Vandermonde determinant, known to be nonzero for distinct sample values $t_{1}, \ldots, t_{s}$. The connection to the Abel equation is made by choosing sample values $x=x_{0}, x=$ $\Phi\left(x_{0}\right)$, and so forth, in the linear combination $c_{1}+c_{2} \alpha(x)+\cdots+c_{s}(\alpha(x))^{s-1}=0$, and then writing the system $A \mathbf{c}=\mathbf{0}$ for vector $\mathbf{c}$ to show $\mathbf{c}=\mathbf{0}$.

The proof that functions (2.12) satisfy the equation proceeds by inserting $f$ into (2.1). The binomial formula $(a+b)^{r}=\sum_{m=0}^{r}\left(\begin{array}{c}r \\ m\end{array}\right) a^{m} b^{r-m}$ is applied to give

$$
\begin{aligned}
\sum_{n=0}^{k} a_{n} f \circ \Phi^{n}(x) & =\sum_{n=0}^{k} a_{n}(\alpha(x)+n)^{r} \lambda_{0}^{\alpha(x)+n} \\
& =\left(\sum_{m=0}^{r}\left(\begin{array}{c}
r \\
m
\end{array}\right)\left(\sum_{n=0}^{k} n^{r-m} a_{n} \lambda_{0}^{n}\right)(\alpha(x))^{m}\right) \lambda_{0}^{\alpha(x)} \\
& =\left(\sum_{m=0}^{r}\left(\begin{array}{c}
r \\
m
\end{array}\right)(0)(\alpha(x))^{m}\right) \lambda_{0}^{\alpha(x)} \\
& =0
\end{aligned}
$$

the last step is justified by Lemma 2.5 .

Independence follows directly from Lemmas 2.6 and 2.7. The details of proof for general $X$ parallel the above steps, essentially replacing $\alpha$ by $X^{*} \alpha$. The proof is complete.

THEOREM 2.8. If the characteristic equation (2.3) has conjugate complex roots $\lambda_{1}=\bar{\lambda}_{2}=$ $r(\cos \omega+i \sin \omega)$, then the corresponding linear homogeneous functional equation possesses two solutions in the form

$$
f_{1}=r^{X^{*} \alpha(x)} \cos \left(\omega X^{*} \alpha(x)\right), \quad f_{2}=r^{X^{*} \alpha(x)} \sin \left(\omega X^{*} \alpha(x)\right) .
$$

The proof is left to the reader. 
6 A remark on linear functional equations

\section{An application}

Let $S$ be the space of all continuous functions which map the interval $(-\infty, \infty)$ one-toone onto itself. Let the canonical function be $X(x)=x$ (identity), then $X^{*}(x)=x$. Let $\Phi(x)=x+1$. The Abel functional equation is then

$$
\alpha(x+1)=\alpha(x)+1
$$

A linear homogeneous functional equation with constant coefficients is

$$
a_{k} f \Phi^{k}(x)+a_{k-1} f \Phi^{k-1}(x)+\cdots+a_{1} f \Phi^{1}(x)+a_{0}, f \Phi^{0}(x)=0
$$

where $f \Phi^{j}(x)$ is a composite function $f\left(\Phi^{j}(x)\right), j=1,2, \ldots, k$, and $\Phi^{j}$ is defined by successive composition. Suppose $\lambda>0$ is a root of the characteristic equation. By the theorems above, it has a solution of the form

$$
f=\lambda^{\alpha(x)}
$$

where $\alpha$ satisfies Abel equation (3.1).

Linear system (3.2) is a linear difference equation with constant coefficients of the form

$$
a_{k} f(x+k)+a_{k-1} f(x+k-1)+\cdots+a_{1} f(x+1)+a_{0} f(x)=0,
$$

because $\Phi(x)=x+1$. Abel equation (3.1) has a solution $\alpha(x)=x$. If $\lambda$ is a positive root of the characteristic equation, then the function $f=\lambda^{x}$ is a solution of (3.4).

3.1. Equivalence of points in $\mathscr{F}$, orbit of a point in $S$. Let $\Phi \in S$. Two points $x, y \in \mathscr{F}$ are $\Phi$-equivalent if and only if there are numbers $\mu, \nu \in \mathbb{Z}$ such that

$$
\Phi^{\mu}(x)=\Phi^{\nu}(y)
$$

The equivalence is reflexive, symmetric, and transitive. It means that there is a decomposition of the set $\mathscr{F}$ into disjoint sets of equivalent points.

The set $O_{\Phi}\left(x_{0}\right)$ of all points $\Phi$-equivalent to a point $x_{0} \in J$ is called the $\Phi$-orbit of the point $x_{0}$.

For the classical difference equation (3.4), in which $X(x)=X^{*}(x)=x$ and $\Phi(x)=$ $x+1$, the orbit of 0 is the set $\mathbb{Z}$ of integers and the orbit of $x_{0}$ is the translate of this set by $x_{0}: O_{\Phi}\left(x_{0}\right)=\left\{x_{0}+\mu\right\}_{\mu=-\infty}^{\infty}$.

LeMma 3.1. Let $x_{0} \in \mathscr{F}, \mu, \nu \in \mathbb{Z}$. Let

$$
x_{\mu}=X^{*} \Phi^{\mu}\left(x_{0}\right)
$$

Then the set of points $\left\{x_{\mu}\right\}_{\mu=-\infty}^{\infty}$ is the $\Phi$-orbit of the point $x_{0}$. 
Proof. Because $\boldsymbol{\Phi}^{0}=X$ and $x_{\mu} \in \mathscr{F}$, then $x_{0}=X^{*} \boldsymbol{\Phi}^{0}\left(x_{0}\right)$. Let $p, q \in \mathbb{Z}$. To show that each two points $x_{p}, x_{q}$ defined by (3.6) are $\Phi$-equivalent in $\mathscr{F}$, we will show that there are numbers $\mu, \nu \in \mathbb{Z}$ such that

$$
\Phi^{\mu}\left(x_{p}\right)=\boldsymbol{\Phi}^{\nu}\left(x_{q}\right) \text {. }
$$

Use formula (3.6) to show that (3.7) is equivalent to

$$
\boldsymbol{\Phi}^{\mu}\left(X^{*}\left(\boldsymbol{\Phi}^{p}\left(x_{0}\right)\right)\right)=\boldsymbol{\Phi}^{\nu}\left(X^{*}\left(\boldsymbol{\Phi}^{q}\left(x_{0}\right)\right)\right) .
$$

The definition of multiplication $\circ$ implies the above is equivalent to

$$
\boldsymbol{\Phi}^{\mu+p}\left(x_{0}\right)=\boldsymbol{\Phi}^{\nu+q}\left(x_{0}\right) .
$$

Choose $\mu=q, \nu=p$ to obtain (3.7).

3.2. Behavior of solutions at infinity. Consider a linear homogeneous $k$ th-order functional equation with constant coefficients (2.1). Let the characteristic equation (2.3) have roots satisfying

$$
\lambda_{1}>\lambda_{2}>\cdots>\lambda_{k}>0
$$

Let $\alpha \in S$ be a solution of the Abel functional equation (2.2) and let $X^{*}$ be the inverse function to canonical function $X$. Assume $c_{1}, \ldots, c_{k}$ are continuous $\Phi$-automorphic functions on $J$, that is, $c_{j}=c_{j} \circ \Phi$. Then a solution of (2.1) is given by

$$
f(x)=c_{1}(x) \lambda_{1}^{X^{*} \alpha(x)}+c_{2}(x) \lambda_{2}^{X^{*} \alpha(x)}+\cdots+c_{k}(x) \lambda_{k}^{X^{*} \alpha(x)}
$$

The right side of (3.11) can be rearranged into the expression

$$
f(x)=\lambda_{1}^{X^{*} \alpha(x)}\left[c_{1}(x)+c_{2}(x)\left(\frac{\lambda_{2}}{\lambda_{1}}\right)^{X^{*} \alpha(x)}+\cdots+c_{k}(x)\left(\frac{\lambda_{k}}{\lambda_{1}}\right)^{X^{*} \alpha(x)}\right] .
$$

The inequalities

$$
0<\frac{\lambda_{j}}{\lambda_{1}}<1, \quad j=2,3, \ldots, k
$$

imply

$$
\lim _{x \rightarrow \infty} c_{j}(x)\left(\frac{\lambda_{j}}{\lambda_{1}}\right)^{X^{*} \alpha(x)}=0
$$

Formally, at least,

$$
\lim _{x \rightarrow \infty} f(x)=\lim _{x \rightarrow \infty} c_{1}(x) \lambda_{1}^{X^{*} \alpha(x)} .
$$


We will discuss the meaning of (3.15). The following situations can occur.

(1) If $\lambda_{1}>1$, then $\lim _{x \rightarrow \infty} f(x)=\infty$, because $\lim _{x \rightarrow \infty} X^{*} \alpha(x)=\infty$.

(2) If $\lambda_{1}=1$, then $\lim _{x \rightarrow \infty} f(x)$ does not generally exist.

(3) If $\lambda_{1}<1$, then $\lim _{x \rightarrow \infty} f(x)=0$, because $\lim _{x \rightarrow \infty} X^{*} \alpha(x)=\infty$. Consider (2.1) on the $\Phi$-orbit of a point $x_{0} \in \mathscr{L}$. Then sequences

$$
\left\{\lambda_{1}^{X^{*} \alpha X^{*} \Phi^{n}\left(x_{0}\right)}\right\},\left\{\lambda_{2}^{X^{*} \alpha X^{*} \Phi^{n}\left(x_{0}\right)}\right\}, \ldots,\left\{\lambda_{k}^{X^{*} \alpha X^{*} \Phi^{n}\left(x_{0}\right)}\right\}
$$

are the solutions evaluated along the $\Phi$-orbit. Because $\alpha \in S$ satisfies Abel functional equation (2.2), then the sequences can be written in the form

$$
\left\{\lambda_{1}^{\alpha\left(x_{0}\right)+n}\right\}_{n=1}^{\infty},\left\{\lambda_{2}^{\alpha\left(x_{0}\right)+n}\right\}_{n=1}^{\infty}, \ldots,\left\{\lambda_{k}^{\alpha\left(x_{0}\right)+n}\right\}_{n=1}^{\infty} .
$$

Therefore, we have a solution formula

$$
f\left(x_{0}+n\right)=c_{1}\left(x_{0}\right) \lambda_{1}^{\alpha\left(x_{0}\right)+n}+c_{2}\left(x_{0}\right) \lambda_{2}^{\alpha\left(x_{0}\right)+n}+\cdots+c_{k}\left(x_{0}\right) \lambda_{k}^{\alpha\left(x_{0}\right)+n} .
$$

Using the ideas above, the limits at infinity are determined as follows.

(1) If $\lambda_{1}>1$, then $\left\{\lambda_{1}^{\alpha\left(x_{0}\right)+n}\right\}$ diverges to $\infty$ and $\lim _{n \rightarrow \infty} f\left(x_{0}+n\right)=\infty$.

(2) If $\lambda_{1}=1$, then $\left\{\lambda_{1}^{\alpha\left(x_{0}\right)+n}\right\}$ is a constant sequence of ones and the sequence $\left\{c_{1}\left(x_{0}\right)\right.$ $\left.\lambda_{1}^{\alpha\left(x_{0}\right)+n}\right\}$ is a constant sequence of numbers $c_{1}\left(x_{0}\right)$. Hence $\lim _{n \rightarrow \infty} f\left(x_{0}+n\right)=$ $c_{1}\left(x_{0}\right)$.

(3) If $0<\lambda_{1}<1$, then the sequence $\left\{c_{1}\left(x_{0}\right) \lambda_{1}^{\alpha\left(x_{0}\right)+n}\right\}$ is a decreasing sequence convergent to zero and $\lim _{n \rightarrow \infty} f\left(x_{0}+n\right)=0$.

Note 3.2. The limit for $x \rightarrow-\infty$ can be determined using the same techniques of analysis.

Note 3.3. The results for behavior of solutions for difference equations can be obtained from the results for (2.1). The idea is to evaluate along the orbit $O_{\Phi}\left(x_{0}\right), x_{0} \in \mathscr{L}$.

\section{References}

[1] S. N. Elaydi, An Introduction to Difference Equations, 2nd ed., Undergraduate Texts in Mathematics, Springer, New York, 1999.

[2] A. O. Gel'fond, Differenzenrechnung, Hochschulbücher für Mathematik, vol. 41, VEB Deutscher Verlag der Wissenschaften, Berlin, 1958.

[3] M. Kuczma, Functional Equations in a Single Variable, Monografie Matematyczne, vol. 46, Państwowe Wydawnictwo Naukowe, Warsaw, 1968.

[4] M. Kuczma, B. Choczewski, and R. Ger, Iterative Functional Equations, Encyclopedia of Mathematics and Its Applications, vol. 32, Cambridge University Press, Cambridge, 1990.

[5] F. Neuman, Funkcionální Rovnice, SNTL, Prague, 1986.

Jitka Laitochová: Mathematical Department, Faculty of Education, Palacký University,

Žižkovo náměsti 5, Olomouc 77140, Czech Republic

E-mail address: jitka.laitochova@upol.cz 